\title{
Fine Structure of the Zeros of Orthogonal Polynomials: A Progress Report
}

\author{
Barry Simon*
}

\begin{abstract}
We consider the asymptotics of zeros of OPRL and POPUC as $n \rightarrow \infty$, focusing on the structure on a scale in $x$ of order $1 / n$. We discuss three recent results (on Poisson behavior in the random case, clock on the a.c. case, and $\beta$-distribution in between) and open questions and speculations.
\end{abstract}

\section{Introduction}

In this paper, we will discuss zeros of orthogonal polynomials. This subject goes back to Gauss' discovery that the best discrete approximations of Riemann integrals involve zeros of Legendre polynomials, and has spawned a huge literature not only among workers on OPs but within the general theoretical and mathematical physics communities who study "eigenvalue statistics." In particular, much of the work on random matrices is connected to this subject.

The general theory has two levels, both going back to a 1940 paper of ErdösTurán [10]. The bulk behavior concerns what fraction of the $n$ zeros of $P_{n}$ lie in a given subset, $S$, of $\mathbb{R}$. The fine structure uses a microscope to look on a scale of size $O\left(\frac{1}{n}\right)$ where, as $n \rightarrow \infty$, there are typically finitely many zeros. The two most heavily studied regimes are where the zeros are Poisson distributed, that is, no correlation between nearby zeros, and where there is rigid equal spacing between neighboring zeros.

Over the past few years, I have written a series of papers on this subject $[38,39,40,21,2]$, including one review $[42]$, but it is time for another review of recent progress and open questions.

We begin by setting notation and terminology. In much of this paper, we study orthogonal polynomials on the real line (OPRL). We start with a measure, $d \mu$, on $\mathbb{R}$ of the form

$$
d \mu(x)=w(x) d x+d \mu_{\mathrm{s}}(x)
$$

where $d \mu_{\mathrm{s}}$ is Lebesgue singular. We will only consider cases where $d \mu$ is compactly supported. See [46] for background on OPRL.

2000 Mathematics Subject Classification. 26C10,65H17,05E35.

Key words and phrases. Orthogonal polynomials, clock behavior, Poisson process.

Supported in part by NSF grant DMS-0652919. 
Given $\mu, P_{n}(x)$ are the monic orthogonal polynomials and $p_{n}(x)$ the orthonormal polynomials. $P_{n}(x)$ has $n$ simple zeros, all on $\mathbb{R}$, indeed on the convex hull of $\operatorname{supp}(d \mu)$. We will be interested in zeros near a fixed $x_{0} \in \mathbb{R}$, which we label $x_{j}^{(n)}\left(x_{0}\right)$, with

$$
\ldots x_{-k}^{(n)}\left(x_{0}\right)<\cdots<x_{-1}^{(n)}\left(x_{0}\right)<x_{0} \leq x_{0}^{(n)}\left(x_{0}\right)<x_{1}^{(n)}\left(x_{0}\right) \ldots
$$

$\mu$ determines, and in turn is determined by, a set of Jacobi parameters, $\left\{a_{n}, b_{n}\right\}_{n=1}^{\infty}$, given by the recursion relations for the $P_{n}$ :

$$
x P_{n}(x)=P_{n+1}(x)+b_{n+1} P_{n}(x)+a_{n}^{2} P_{n-1}(x)
$$

Here $a_{n}>0, b_{n} \in \mathbb{R}$, and there is a one-one correspondence between uniformly bounded Jacobi parameters and nontrivial probability measures of compact support.

We will also consider orthogonal polynomials on the unit circle (OPUC) and paraorthogonal polynomials (POPUC). See $[\mathbf{3 6}, \mathbf{3 7}]$ for a discussion of OPUC and $[4,43,53]$ for POPUC.

The OPUC are determined by $\mu$ with monic polynomials, $\Phi_{n}(z)$, and orthonormal, $\varphi_{n}(z)$. The recursion relations now have the Szegö form

$$
\Phi_{n+1}(z)=z \Phi_{n}(z)-\bar{\alpha}_{n} \Phi_{n}^{*}(z)
$$

where

$$
\Phi_{n}^{*}(z)=z^{n} \overline{\Phi_{n}(1 / \bar{z})}
$$

The $\alpha_{n} \in \mathbb{D}$ are called Verblunsky coefficients and there is a one-one correspondence between $\left\{\alpha_{n}\right\}_{n=0}^{\infty} \in \mathbb{D}^{\infty}$ and nontrivial probability measures on $\partial \mathbb{D}$.

The POPUC are defined by $\left\{\alpha_{j}\right\}_{j=0}^{n-2} \in \mathbb{D}^{n-1}$ and $\alpha_{n-1} \in \partial \mathbb{D}$ by (1.4). The new element is $\alpha_{n-1} \in \partial \mathbb{D}$, not in $\mathbb{D}$. OPUC have all their zeros in $\mathbb{D}$. POPUC have their zeros in $\partial \mathbb{D}$ and are simple, and so similar to OPRL.

The bulk behavior of the zeros is described by defining a probability measure, $d \nu_{n}$, which assigns a weight $1 / n$ to each zero of $P_{n}$ in the OPRL case or, in the POPUC case, $\Phi_{n}$. In the OPUC case, zeros can have multiplicity $k>1$ and $d \nu_{n}$ gives such zeros a weight $k / n$. We say that the density of zeros (or density of states) exists if $d \nu_{n}$ has a weak limit, $d \nu$. It often happens that $d \nu$ is the equilibrium measure for $\operatorname{supp}(d \mu)$ (see $[47,41])$ and even more generally that for some $L^{1}(\mathbb{R}, d x)$ function,

$$
d \nu(x)=\rho(x) d x
$$

When one turns to fine structure, two regimes have received the most attention-indeed, until very recently, all the attention. One is the regime of truly random recursion parameters where the distribution of zeros, after scaling distances by $n$ about a point $x_{0}$, is a Poisson process. The key early papers are by Molchanov [32] and Minami [31].

The other extreme is the nice a.c. spectrum region where it is often known that one has clock behavior

$$
x_{j+1}^{(n)}\left(x_{0}\right)-x_{j}^{(n)}\left(x_{0}\right) \sim \frac{1}{n \rho\left(x_{0}\right)}
$$

Here the key works are Erdös-Turán [10], Freud [12], and Deift et al. [9], and this is the main area I studied in $[38,39,40,21]$. Freud realized a connection of zeros and the asymptotics of the CD kernel slightly off diagonal, so we recall 
the definition and some properties of the kernel. The Christoffel-Darboux kernel is defined for $x, y \in \mathbb{R}$ by

$$
K_{n}(x, y)=\sum_{j=0}^{n} p_{j}(x) p_{j}(y)
$$

The connection to zeros depends on the CD formula

$$
K_{n}(x, y)=\frac{a_{n+1}\left(p_{n+1}(x) p_{n}(y)-p_{n+1}(y) p_{n}(x)\right)}{x-y}
$$

For a review of the CD kernel, see [44].

One aspect of CD kernel asymptotics is "classical." It involves the notion that the diagonal asymptotics is often given by

$$
\frac{1}{n} K_{n}(x, x) \sim \frac{\rho(x)}{w(x)}
$$

a notion associated to work of Freud and Nevai, discussed by Nevai in [33]. This Freud-Nevai vision was realized especially in Máté-Nevai-Totik [30] and Totik [49].

A revolution in establishing off-diagonal asymptotics came from two remarkable papers of Lubinsky $[\mathbf{2 7}, \mathbf{2 5}$, part of a series that also includes $[\mathbf{2 2}, \mathbf{2 3}, \mathbf{2 4}, \mathbf{2 6}]$ and several other papers. In particular, his second approach in [25] will play a major role below.

Before leaving the subject of CD kernels, we want to recall the second kind polynomials, $q_{n}(x)$, defined as follows. Let $\tilde{p}_{n}(x)$ be the OPRL associated to oncestripped Jacobi parameters, that is, to $\left\{\tilde{a}_{n}, \tilde{b}_{n}\right\}_{n=1}^{\infty}$ where

$$
\tilde{a}_{n}=a_{n+1} \quad \tilde{b}_{n}=b_{n+1}
$$

Then

$$
q_{n}(x)=a_{1}^{-1} \tilde{p}_{n}(x)
$$

We will need the associated CD kernel

$$
K_{n}^{(q)}(x, y)=\sum_{j=0}^{n} q_{j}(x) q_{j}(y)
$$

Section 2 describes "older" results, including Lubinsky's first approach [27] to the off-diagonal CD kernel. In particular, it describes in some detail Poisson and clock behavior. Section 3 discusses three recent significant results on the Poisson, intermediate, and clock regimes. Section 4 discusses open questions.

It is pleasure to thank Paco Marcellán and Andrei Martinez-Finkelstein for the invitation to speak at the IWOPA'08 conference and Bill López for the excuse he gave us for the conference (and for his many signal contributions). I would like to thank Jonathan Breuer, Rowan Killip, Nikolai Makarov, Andrei MartinezFinkelshtein, Eric Ryckman, Mihai Stoiciu, and Vilmos Totik for useful discussions.

\section{The Past Is Prologue}

Here we will recall some results that describe the two main types of reasonably well-understood fine structure. We begin with Stoiciu's results [48] on Poisson behavior for random POPUC (recalling that Molchanov [32] and Minami [31] were the pioneers in Poisson behavior for OPs): 
THEOREM 2.1 (Stoiciu $[\mathbf{4 8}]$ ). Fix $R<1$. Let

$$
\Omega=\left[\begin{array}{l}
\infty \\
\times
\end{array}\{z \in \mathbb{C}|| z \mid \leq R\}\right] \times \partial \mathbb{D}
$$

and let $d \eta$ be the measure on $\Omega$ describing independent, identically distributed random variables, uniformly distributed on $\{z|| z \mid \leq R\}$ with $\omega_{\infty}$ independent and uniformly distributed on $\partial \mathbb{D}$. For $\omega \in \Omega$, let $\Phi_{n}(z ; \omega)$ be the POPUC with $\alpha_{j}=\omega_{j}, j=0, \ldots, n-2 ; \alpha_{n-1}=\omega_{\infty}$. Then for any $z_{0}=e^{i \theta_{0}} \in \partial \mathbb{D}$ and any $a_{1}<b_{1}<\cdots<a_{k}<b_{k}$ fixed,

$$
\begin{gathered}
\lim _{n \rightarrow \infty} \operatorname{Prob}\left\{\Phi_{n}(z ; \omega) \text { has exactly } m_{j} \text { zeros in } \theta \in\left[\theta_{0}+\frac{2 \pi a_{j}}{n}, \theta_{0}+\frac{2 \pi b_{j}}{n}\right],\right. \\
j=1, \ldots, k\}=\prod_{j=1}^{k}\left[\frac{\left(b_{j}-a_{j}\right)^{m_{j}}}{m_{j} !} e^{-\left(b_{j}-a_{j}\right)}\right]
\end{gathered}
$$

REMARKS. 1. What is critical is rotation invariance of the individual distribution, not the exact form.

2. For related results on the zeros of the OPUC in this case, see Davies-Simon [8].

Two things are critical in this proof:

(a) Exponential localization of the eigenstates, which implies asymptotic independence of zeros produced by subboxes.

(b) An estimate that assures the probability of two zeros in an interval of size $2 \pi \varepsilon / n$ is $o(\varepsilon)$.

The best results on clock spacing for OPRL with spectrum $[-2,2]$ is

THEOREM 2.2 (Lubinsky $[\mathbf{2 7}, \mathbf{2 2}]$ ). Let $d \mu$ be a measure supported on $[-2,2]$ which is regular. Let $[a, b]$ be an interval in $[-2,2]$ so that on $[a, b]$,

$$
d \mu(x)=w(x) d x
$$

with $w$ continuous and strictly positive. Define $x_{j}^{(n)}\left(x_{0}\right)$ by (1.2). Then for any $x_{0} \in(a, b)$,

$$
n\left(x_{j+1}^{(n)}\left(x_{0}\right)-x_{j}^{(n)}\left(x_{0}\right)\right) \rightarrow \frac{1}{\rho\left(x_{0}\right)}
$$

where $\rho\left(x_{0}\right)$ is given by (1.6).

REMARKs. 1. Regularity here means $\left(a_{1} \ldots a_{n}\right)^{1 / n} \rightarrow 1$; see $[47,41]$.

2. (2.3) is called clock behavior.

3. It is known $[\mathbf{1 1}, \mathbf{2 7}, \mathbf{4 5}, \mathbf{5 1}]$ that the three conditions, $w(x)$ continuous and nonvanishing and $d \mu_{\mathrm{s}}=0$, can be replaced by the three conditions of local Szegö condition, $x_{0}$ being a Lebesgue point for $w$, and $\lim _{k \rightarrow \infty} k \mu_{\mathrm{s}}\left(x_{0}-\frac{1}{k}, x_{0}+\frac{1}{k}\right)=0$. Also, $\operatorname{supp}(d \mu)=[-2,2]$ can be replaced by an essential support requirement.

Lubinsky's approach is related to proving universality. Let $K_{n}$ be the CD kernel given by (1.8). Universality at $x_{0}$ says (uniformly in $|a|<A,|b|<A$ for each $A<\infty$ )

$$
\lim _{n \rightarrow \infty} \frac{K_{n}\left(x_{0}+\frac{a}{n}, x_{0}+\frac{b}{n}\right)}{K_{n}\left(x_{0}, x_{0}\right)}=\frac{\sin \left(\pi \rho\left(x_{0}\right)(b-a)\right)}{\pi \rho\left(x_{0}\right)(b-a)}
$$


Since $\sin (\pi(c-d))=0 \Leftrightarrow c-d \in \mathbb{Z}$ and the CD formula, (1.9), says that if $p_{n}\left(x_{1}\right)=0$, then $p_{n}\left(x_{2}\right)=0$ for $x_{2} \neq x_{1}$ if and only if $K_{n}\left(x_{1}, x_{2}\right)=0$, (2.4) should be connected to clock spacing. Indeed,

Theorem 2.3 (Freud-Levin Theorem). Universality, (2.4), at $x_{0}$ implies clock spacing, (2.3).

REMARKS. 1. This result is implicit in Freud [12] who proved universality for sufficiently nice measures on $[-2,2]$. It was rediscovered by Levin and reported in Levin-Lubinsky [22].

2. Under some additional growth assumptions on $K_{n}$, clock spacing implies universality; see, for example, [23].

The same argument relates two weaker sets of notions. The striking thing about clock behavior is equal spacing, which is independent of the spacing multiplied by $n$ having a limit. We say quasi-clock behavior holds at $x_{0}$ if for any fixed $j \in \mathbb{Z}$ as $n \rightarrow \infty$,

Define

$$
\frac{x_{j+1}^{(n)}\left(x_{0}\right)-x_{j}^{(n)}\left(x_{0}\right)}{x_{1}^{(n)}\left(x_{0}\right)-x_{0}^{(n)}\left(x_{0}\right)} \rightarrow 1
$$

$$
\rho_{n}\left(x_{0}\right)=\frac{1}{n} w\left(x_{0}\right) K_{n}\left(x_{0}, x_{0}\right)
$$

We say weak universality holds if

$$
\frac{K_{n}\left(x_{0}+\frac{a}{n \rho_{n}}, x_{0}+\frac{b}{n \rho_{n}}\right)}{K_{n}\left(x_{0}, x_{0}\right)} \rightarrow \frac{\sin (\pi(b-a))}{\pi(b-a)}
$$

TheOREM 2.4 (Weak Freud-Levin). Weak universality at $x_{0}$ implies quasi-clock behavior at $x_{0}$.

If

$$
\rho_{n}\left(x_{0}\right) \rightarrow \rho\left(x_{0}\right)
$$

then weak universality implies universality. This links up fine structure to the Freud-Nevai vision discussed in Section 1.

Lubinsky proved Theorem 2.2 by using a model measure for which universality holds, and then used a clever comparison argument. He used Legendre polynomials as his model, but the calculations are more explicit and easier for Chebyshev polynomials of either the first or second kind.

Lubinsky's result was extended to general compact subsets, $\mathfrak{e}$, of $\mathbb{R}$ by Findley [11], Simon [45], and Totik [51]. Findley and Totik use the method of polynomial mappings and approximation $[49,50]$. Simon uses approximation and Jost functions for the isospectral torus as a model.

One important aspect of the second approach of Lubinsky [25], discussed in the next section, is that it goes beyond the need for a model to compare to.

\section{The Present: Three Breakthroughs}

We want to focus on three recent major results that deal with three different regimes of zeros:

(1) The result of Combes, Germinet, and Klein [7] on Poisson statistics for multidimensional Schrödinger operators.

(2) The results of Killip and Stoiciu [15] on decaying random models. 
(3) The new approach of Lubinsky [25] and the associated work of Avila, Last, and Simon [2] on clock spacing.

We will say very little about (1) since it is peripheral to our concerns here and a lot about (3) since it was the content of my talk at the conference for which this is the proceedings.

The bulk of this review concerns OPs which arise from one-dimensional difference equations. Combes-Germinet-Klein [7] study the continuum differential Schrödinger operator, $-\Delta+V$, in higher dimension. While the initial results on Poisson statistics [32] were on continuum models, they were definitely onedimensional. Minami [31] could handle arbitrary dimensions, but his models were discrete. In part, he had one key result (the Minami lemma) that seemed to be restricted to rank one perturbations. Extending and understanding this (done by Bellissard-Hislop-Stolz [3], Combes-Germinet-Klein [6], and Graf-Vaghi [13]) was a first step-but even with it, the full argument in [7] is a subtle piece of work that settles a problem that has been open for more than fifteen years.

Killip-Stoiciu [15] discuss POPUC with random decaying Verblunsky coefficients. They require rotation invariance, so to simplify exposition, we will suppose the distributions are over suitable disks. Thus, we suppose $A_{n}(\omega)$ are iidrv with individual distribution uniform over the disk of radius $\frac{1}{2} . c_{0}, c_{1}, \ldots$ will be a sequence with $0<c_{j} \leq 1$ and we take

$$
\alpha_{n}(\omega)=c_{n} A_{n}(\omega)
$$

For background, we recall the following from [37]:

THEOREM 3.1. Let $\alpha_{n}$ obey (3.1) where $c_{n}<1$ and for some $N_{0}$ and $n>N_{0}$, $0<\gamma<1$,

$$
c_{n}=k n^{-\gamma}
$$

Then, with $d \mu_{\omega}$ the measure with Verblunsky coefficients $\alpha_{n}(\omega)$,

(i) If $\gamma>\frac{1}{2}$ for a.e. $\omega, d \mu_{\omega}$ is purely absolutely continuous with support $\partial \mathbb{D}$ and for Lebesgue a.e. $\theta$, bounded transfer matrix at $z=e^{i \theta}$.

(ii) If $\gamma<\frac{1}{2}$, for a.e. $\omega, d \mu_{\omega}$ is pure point with eigenvalues dense in $\partial \mathbb{D}$ and eigenvectors decaying as fast as $\exp \left(-d n^{1-2 \gamma}\right)$.

(iii) For $\gamma=\frac{1}{2}$, if $k^{2}>8$, for a.e. $\omega, d \mu_{\omega}$ is dense pure point with polynomially decaying eigenfunctions. If $k^{2} \leq 8, d \mu_{\omega}$ is purely singular continuous with constant Hausdorff dimension $1-\frac{1}{8} k^{2}$.

REMARKs. 1. $\left\langle\left|A_{n}(\omega)\right|^{2}\right\rangle=\frac{1}{8}$, so the $\Gamma$ of $(12.7 .11)$ of $[37]$ for $\gamma=\frac{1}{2}$ is $\Gamma=\frac{k}{\sqrt{8}}$.

2. Constant Hausdorff dimension, $d$, means $\mu_{\omega}(A)=0$ for any set of dimension smaller than $d$, and $\mu_{\omega}$ is supported on a set of dimension $d$.

Killip-Stoiciu have results on the zeros that have a similar three-part breakdown. For POPUC, we need a phase, $\alpha_{n-1} \in \partial \mathbb{D}$, which we take random, uniform on $\partial \mathbb{D}$, and independent of $\alpha_{j}(\omega)$ :

THEOREM 3.2 (Killip-Stoiciu [15]). Under the hypotheses of Theorem 3.1,

(i) For $\gamma>\frac{1}{2}$, for a.e. $\omega$ and $z_{0}$, the zeros of the POPUC have clock spacing with density $2 \pi / n$ in the sense that if $z_{0}=e^{i \theta_{0}}$ and $\theta_{j}^{(n)}\left(\theta_{0}\right)$ are defined by $\ldots<\theta_{-1}^{(n)}<\theta_{0} \leq \theta_{n}^{(n)}<\theta_{-2}^{(n)}<\ldots$, so $e^{i \theta_{j}^{(n)}\left(\theta_{0}\right)}$ are all the zeros of $\Phi^{\left(\alpha_{n}\right)}(z)$ near $z_{0}$, then

$$
n\left(\theta_{j+1}^{(n)}-\theta_{j}^{(n)}\right) \rightarrow 2 \pi
$$


as $n \rightarrow \infty$.

(ii) For $\gamma<\frac{1}{2}$, the zeros of the POPUC are locally Poisson distributed in the sense of Theorem 2.1.

(iii) If $\gamma=\frac{1}{2}$, the zeros asymptotically have a $C \beta E$ distribution where

$$
\beta=\frac{16}{k^{2}}
$$

By a finite $\mathrm{C} \beta \mathrm{E}$ (for circular beta ensemble) distribution, we mean the measure on $(\partial \mathbb{D})^{n}$ given by the density for $\left(e^{i \theta_{1}}, \ldots, e^{i \theta_{n}}\right)$

$$
N_{\beta, n}^{-1} \prod_{j<k}\left|\theta_{j}-\theta_{k}\right|^{\beta}
$$

where $N_{\beta, n}$ is a normalization constant. The values $\beta=1,2,4$ correspond to orthogonal, unitary, and symplectic ensembles, and the properties of the limit (with $1 / n$ scaling) are understood in that there are explicit formulae for $n$-point functions. For other $\beta$, the understanding is much less complete. (iii) means the probabilities of zeros of the POPUC and the points of $\mathrm{C} \beta \mathrm{E}$ are asymptotically equal. The most difficult part, namely (iii), relies on earlier work of Killip-Nenciu [14] who identified the distribution of Verblunsky coefficients for $\mathrm{C} \beta \mathrm{E}$ and a subtle convergence argument.

Finally, we turn to describing the work of Lubinsky [25] and Avila-Last-Simon [2]. Here is a general result in ALS:

THEOREM $3.3([2])$. Let $d \mu$ be a measure on $\mathbb{R}$ with compact support. Let $\Sigma_{0}$ be a set of positive Lebesgue measure so that

(i) For a.e. $x_{0} \in \Sigma_{0}, w\left(x_{0}\right)>0$.

(ii) For a.e. $x_{0} \in \Sigma_{0}$,

$$
\frac{1}{n+1} K_{n}\left(x_{0}, x_{0}\right) \rightarrow \frac{\rho_{\infty}\left(x_{0}\right)}{w\left(x_{0}\right)}>0
$$

(iii) For a.e. $x_{0} \in \Sigma$ with $K_{n}^{(q)}$ given by (1.13),

$$
\sup _{n} \frac{1}{n+1} K_{n}^{(q)}\left(x_{0}, x_{0}\right)<\infty
$$

Then for a.e. $x_{0} \in \Sigma$, universality (and so, quasi-clock behavior) holds at $x_{0}$.

REMARK. $\rho_{\infty}$ is not assumed to be the density of a density of zeros. Rather, it is defined by (3.5), that is, condition (ii) is a statement that the limit exists (and is finite and nonzero).

Furthermore, [2] shows the hypotheses hold in the ergodic case. Let $(\Omega, d \eta(\omega))$ be a probability measure space where $\Omega$ is a compact metric. Let $T: \Omega \rightarrow \Omega$ be continuous and invertible and ergodic for $d \eta$. Let $A, B$ be continuous functions on $\Omega$ where $A$ has values in $(0, \infty)$ and $B$ in $\mathbb{R}$. To each $\omega \in \Omega$, we define a Jacobi matrix with parameters

$$
a_{n}(\omega)=A\left(T^{n-1} \omega\right) \quad b_{n}(\omega)=B\left(T^{n-1} \omega\right)
$$

A canonical example is the almost Mathieu equation where $\lambda \in(0, \infty)$ and $\alpha \in \mathbb{R} \backslash \mathbb{Q}$ are fixed, $\Omega=\partial \mathbb{D}, d \eta=d \theta / 2 \pi, T\left(e^{i \theta}\right)=e^{i(\theta+\pi \alpha)}, A\left(e^{i \theta}\right) \equiv 1, B\left(e^{i \theta}\right)=$ $2 \lambda \cos (\theta)\left(\right.$ so $\left.b_{n}\left(e^{i \theta_{0}}\right)=2 \lambda \cos \left(\pi \alpha n+\theta_{0}\right)\right)$. This model is known to have purely a.c. spectrum if $0<\lambda<1$. 
THEOREM $3.4([\mathbf{2}])$. For any ergodic Jacobi matrix with $\left|\Sigma_{\mathrm{ac}}\right|>0$, for a.e. $\omega \in \Omega$ and a.e. $x_{0} \in \Sigma_{\mathrm{ac}}$;

$$
\lim _{n \rightarrow \infty} w\left(x_{0}\right) \frac{1}{n+1} K_{n}\left(x_{0}, x_{0}\right)=\rho_{\infty}\left(x_{0}\right)
$$

where $\rho_{\infty}\left(x_{0}\right)$ is the density of the a.c. part of the density of zeros.

REMARKS. 1. Ergodicity implies the density of zeros exists. It is a result of Kotani [18] (see Simon [35] for the discrete case) that

$$
\Sigma_{\mathrm{ac}}=\{x \mid \gamma(x)=0\}
$$

where $\gamma$ is the Lyapunov exponent, and of Kotani [19] that on this set, the a.c. part of the density of zeros, $\rho_{\infty}(x)$, is exactly the average of weights, $w$.

3. For regular measures with local Szegó conditions on the weight (false when $\Sigma_{\mathrm{ac}}$ is a Cantor set), results like (3.8) are known due to Máté-Nevai-Totik [30] and Totik [49].

Here we want to emphasize the ideas of Lubinsky [25] that get the kernel $\sin (\pi x) / \pi x$. The following is essentially implicit in his paper:

THEOREM 3.5. Let $f(z)$ be an entire function obeying

(i) $\int_{-\infty}^{\infty}|f(x)|^{2} d x \leq 1$.

(ii) $f(0)=1 ;|f(x)| \leq 1$ on $\mathbb{R}$.

(iii) For constants $C$ and $A,|f(z)| \leq C e^{A|z|}$ for all $z \in \mathbb{C}$.

(iv) $f$ is real on $\mathbb{R}$; all zeros of $f$ lie on $\mathbb{R}$.

(v) If $\ldots x_{-n}<\cdots<x_{-1}<0<x_{1}<\cdots<x_{n}<\ldots$ are all the zeros of $f$, then

$$
\left|x_{j}\right| \geq|j|-1
$$

Then

$$
f(z)=\frac{\sin \pi z}{\pi z}
$$

REMARKS. 1. Lubinsky uses stronger hypotheses. In this form, it appears in [2]. [2] can replace (iii) by a weaker hypothesis if (3.10) is replaced by $\left|x_{j}-x_{k}\right| \geq$ $|j-k|-1$.

The ALS proof of this result proceeds by using (iii) to get a Hadamard factorization

$$
f(z)=e^{\alpha z} \prod_{\substack{j \in \mathbb{Z} \\ j \neq 0}}\left(1-\frac{z}{x_{j}}\right) e^{z / x_{j}}
$$

with $\alpha$ real. Thus,

$$
|f(i y)|^{2} \leq \prod_{j \neq 0}\left(1+\frac{y^{2}}{x_{j}^{2}}\right)
$$

which, by (v) and the Euler formula for $\sinh (x)=x \prod_{n=1}^{\infty}\left(1+\frac{x^{2}}{n^{2}}\right)$, leads to

$$
|f(i y)| \leq C_{\varepsilon} e^{(\pi+\varepsilon)|y|}
$$

Phragmén-Lindelöf, (3.14), and $f$ bounded on $\mathbb{R}$ yields

$$
|f(x+i y)| \leq C_{\varepsilon} e^{(\pi+\varepsilon)|y|}
$$

Thus, by the Paley-Wiener theorem, $\hat{f}(k)$, the Fourier transform of $f$, is supported in $[-\pi, \pi]$. (i), (ii), and the Schwarz inequality imply that $\hat{f}(k)$ is $(2 \pi)^{-1 / 2}$ 
times the characteristic function of $[-\pi, \pi]$. The Fourier transform of this characteristic function is $\sin (\pi z) / \pi z$.

3. Lubinsky appeals to various results on the $\sin (z) / z$ kernel, but the proofs of these results depend on Paley-Wiener as above.

Lubinsky [25] used this result to prove

THEOREM 3.6 ([25]). Suppose $d \mu$ has a weight, $w$, and an interval, $I$, so that (i)

$$
\inf _{x \in I} w(x)>0
$$

(ii) For $x_{0} \in I^{\text {int }}$, we have that $x_{0}$ is a Lebesgue point of $d \mu$ in that $\lim _{\delta \downarrow 0}(2 \delta)^{-1} \int\left|w(x)-w\left(x_{0}\right)\right| d x=0$ and $\lim _{\delta \downarrow 0}(2 \delta)^{-1} \mu_{\mathrm{s}}\left(x_{0}-\delta, x_{0}+\delta\right)=0$.

(iii) For a real,

$$
\frac{K_{m}\left(x_{0}+\frac{a}{n}, x_{0}+\frac{a}{n}\right)}{K_{n}\left(x_{0}, x_{0}\right)} \rightarrow 1
$$

uniformly in $|a| \leq A$ for any $A>0$.

Then weak universality (and so, quasi-clock behavior) holds at $x_{0}$.

REMARK. (3.16) is called the Lubinsky wiggle condition.

Lubinsky gets his result by fixing $a \in \mathbb{R}$ and considering limit points, $f(z)$, of

$$
\frac{K_{n}\left(x_{0}+\frac{a}{n \rho_{n}}, x_{0}+\frac{a+z}{n \rho_{n}}\right)}{K_{n}\left(x_{0}, x_{0}\right)}
$$

By Montel's theorem and the a priori bound,

$$
\limsup _{n \rightarrow \infty} \frac{1}{n}\left|K_{n}\left(x_{0}+\frac{w}{n}, x_{0}+\frac{z}{n}\right)\right| \leq C e^{A(|z|+|w|)}
$$

(which follows from the Schwarz inequality and the case $w=z$ which one gets from (3.15) and the Christoffel variational principle [44]) limit points exist, and if all limit points are $\sin (\pi x) / \pi z$, then the limit exists.

Condition (i) of Theorem 3.5 follows from the use of

$$
\int K_{n}(x, y) K_{n}(y, x) d \mu(y)=K_{n}(x, x)
$$

and the use of Lebesgue points (and $\rho_{n}$ scaling). Condition (ii) follows from the Lubinsky wiggle condition (3.16). Condition (iii) follows from (3.18). Condition (iv) follows from properties of the CD kernel [44]. Condition (v) follows from a clever argument of Lubinsky using the Markov-Stieltjes inequalities [44] and the wiggle condition.

In his paper, Lubinsky could only prove the wiggle condition in situations where Totik's methods $[49, \mathbf{5 0}]$ hold and where Totik already used Lubinsky's first method ([51]). But Lubinsky expressed his belief (vindicated by [2]) that the wiggle condition could be proven in other cases.

In proving Theorem 3.3, [2] cannot use (3.15) and a comparison argument to get (3.18) since (3.15) is not assumed. Instead, they use a general perturbation bound that gets exponential bounds from boundedness of the Cesàro averaged transfer matrix $\frac{1}{n+1} \sum_{j=0}^{n}\left\|T_{j}\left(x_{0}\right)\right\|^{2}$ (Theorem 3 of [2]). They get the wiggle condition from the assumed existence (3.5), a use of Egoroff's theorem, and an equicontinuity result for $K_{n}\left(x_{0}+\frac{a}{n}, x_{0}+\frac{a}{n}\right)$ that follows from (3.18). 
To get Theorem 3.4, [2] prove the a.e. existence of the limit in (3.8) by a use of the ergodic theorem (which is subtle since $w\left(x_{0}\right)$ is $\omega$-dependent) and separate arguments that prove the limit is $\omega$-independent, and then that it is $\rho_{\infty}\left(x_{0}\right)$.

\section{The Future: Open Questions and Speculations}

This section has two parts. First, we discuss questions left open in each of the three limit regions we partially understand: Poisson, clock, and $\beta$-distributed. Then we speculate about other singular continuous situations.

Poisson Questions. With regard to Poisson behavior, one especially interesting question is the following:

QUESTION 4.1. Is it true in the Anderson case (OPRL, with $a_{n} \equiv 1$ and $b_{n}$ independent, identically distributed random variables (iidrv) with nice density) that asymptotically as $n \rightarrow \infty$, for any $x_{0} \neq x_{1}$, \{number of zeros of $p_{n}(x)$ in $\left[x_{0}+\right.$ $\left.\left.\frac{a}{n}, x_{0}+\frac{b}{n}\right]\right\}$ and $\left\{\right.$ number of zeros of $p_{n}\left(x_{0}\right)$ in $\left.\left[x_{1}+\frac{c}{n}, x_{1}+\frac{d}{n}\right]\right\}$ are independent? Here $a<b$ and $c<d$. If $x_{0}=x_{1}$ and $a<b<c<d$, this independence is part of what is known as Poisson behavior. Intuitively, independence of nearby $O\left(\frac{1}{n}\right)$ boxes would seem less likely than distant boxes, and one expects the answer to this question is yes. But it is open.

Question 4.2. Prove the analog of the Killip-Stoiciu Poisson result for OPRL. That is, if $a_{n} \equiv 1$ and $b_{n}=c_{n} A_{n}(\omega)$ where $A_{n}$ are iid, say uniformly distributed on $[-1,1]$, and $c_{n}=k n^{-\gamma}$ for $\gamma<\frac{1}{2}$, then zeros are locally Poisson distributed. We do not expect this to be hard.

QUESTION 4.3. What is the fine structure of the eigenvalues of the Anderson model near the edges of the spectrum? This is not here because we necessarily expect the answer to have anything to do with Poisson, but because this subsection is really on situations where one has eigenfunctions decaying at least as fast as $\exp \left(-n^{\alpha}\right)$ for some $\alpha>0$. This question is asking for refinements of Lifschitz tails (see [16] for a discussion of Lifschitz tails). The basic questions are how big an interval at the top of the spectrum do you need to get $O(1)$ for the expected number of zeros, and what are their statistics.

Question 4.4. Poisson behavior is a statement about probabilities. Since probabilities on $O\left(\frac{1}{n}\right)$ are not constant, on that scale there is not almost sure behavior, but there may be almost sure behavior on larger scales. For example, one might expect almost surely on a $(1 / \sqrt{n})$ scale that the fraction of neighboring pairs of eigenvalues with separations in $\left[0, \frac{c}{n}\right]$ is given by the expected number in the Poisson process. What can be said about such almost sure behavior?

Clock Questions. The most interesting open question is a conjecture of [2].

Question 4.5. Prove the following conjecture of Avila, Last, and Simon [2]: Consider a probability measure of compact support given by (1.1). For Lebesgue a.e. $x$ in $\{x \mid w(x)>0\}$, one has quasi-clock behavior. [41] has an example where $\operatorname{supp}(d \mu)=[-2,2],\{x \mid w(x)>0\}=[-2,0], d \mu$ has dense mass points in $[0,2]$, and the density of zeros does not exist. Indeed, both the equilibrium measures for $[-2,0]$ and $[-2,2]$ are limit points of the density of zeros. Thus, clock behavior does not hold (and $\rho_{n}(x)$ does not have a limit). But weak universality can hold-and I believe it does. I suspect that any proof for this special case will allow a treatment of the general conjecture. 
QUESTION 4.6. What can be said about edge behavior in the a.c. ergodic case? Consider first the deterministic case in a single interval. Suppose $a, b \in \operatorname{supp}(d \mu)$ but $(a, b)$ is disjoint from this support. One cannot have clock behavior at $a$ since $(a, b)$ has at most one eigenvalue. For the edge of measures on $[-1,1]$ with Jacobi weight asymptotics at an edge, Lubinsky $[24,26]$ has proven Bessel kernel behavior rather than sin kernel. It is reasonable to guess that unlike bulk behavior which is universal across the a.c. ergodic case, edge behavior is not. In particular, for the almost Mathieu equation at $0<|\lambda|<1$, I would guess that if $\alpha$ has good Diophantine properties, the edge behavior is the same as for Chebyshev of the first kind (i.e., the whole-line free Jacobi matrix). But if $\alpha$ is a Liouville number, the behavior is different-most likely different on different scales.

QUESTION 4.7. Prove the OPRL analog of the Killip-Stoiciu [15] result in the a.c. region, namely if $a_{n} \equiv 1, b_{n}=c_{n} A_{n}(w)$, where $A_{n}$ are as in Question 4.2 , with $c_{n}=k n^{-\gamma}$ and $1 \geq \gamma>\frac{1}{2}$, then one has clock behavior for a.e. $\omega$. Given prior work on this case $([\mathbf{1 7}])$, this should be straightforward. The result for $\gamma>1$ follows from $[21]$.

$\beta$-distribution, Questions. With regard to $\beta$-distributions, we pose the analog of Questions 4.2 and 4.7, namely

QUESTION 4.8. Determine the asymptotic local zero distribution of OPRL with $a_{n} \equiv 1, b_{n}=c_{n} A_{n}(w)$, and $c_{n}=k n^{-1 / 2}$. Now the rate of polynomial decay of eigenfunctions and local Hausdorff dimension [17] is a function of both $k$ and $x$. Presumably that is true of the $\beta$ in the $\beta$-distribution. We have no good tools for identifying $\beta$-distributions directly ( $[\mathbf{1 5}]$ use the known Verblunsky coefficients of Haar measure), so this seems difficult.

Speculations on Singular Spectrum. Singular spectrum is like Tolstoy's remark on dysfunctional families-singular spectrum is unusual in many ways, but that's precisely the point - each is unusual in its own manner. The one example we understand, that of [15], has power decaying eigenfunctions and spectrum (i.e., closed support of the measure) which is an interval. But there are also examples where the support is a closed set of measure zero. Here are two examples:

QUeSTION 4.9. Let $d \mu$ be the classical Cantor measure on the middle third sets. What can be said about the $a_{n}$ 's and about the local structure of the zeros?

Question 4.10. Consider the almost Mathieu equation at the critical coupling, $\lambda=1$. It is known (see Last [20]) that for irrational frequencies, the spectrum is singular continuous and of measure zero. What can be said about the global and the local structure of the zeros?

Let us expand on these two examples. It is known (see, e.g., Makarov [28, 29]) that the potential theory equilibrium measure for the classical Cantor set lives on a set of Hausdorff dimension strictly smaller than the dimension of the Cantor set itself. Totik (private communication) has informed me that his methods with Stahl [47] allow one to prove that this equilibrium measure is the density of zeros measure for this case.

Here is a bold, probably foolhardy, speculation: Perhaps in this case, these zeros are, a.e. with respect to the equilibrium measure, quasi-clock distributed. 
The spacing though, rather than $O\left(\frac{1}{n}\right)$, is $O\left(\frac{1}{n^{1 / d}}\right)$ where $d$ is the dimension of the support of the equilibrium measure.

For this case, because of reflection symmetry about $x=\frac{1}{2}$, we have $b_{n} \equiv \frac{1}{2}$. Motivated by the finite gap case (see $[52, \mathbf{1}, \mathbf{3 4}, \mathbf{5}]$ ), I would conjecture that the $a_{n}$ 's are almost periodic with frequency module determined by the harmonic measures of the subsets of the Cantor set between two gaps.

Motivated by these aspects of the density of zeros for the Cantor measure:

QUESTION 4.11. Does the density of zeros for the critical almost Mathieu model live on a set of smaller local Hausdorff dimension than the spectrum?

As discussed in [41], for noncritical coupling, the density of zeros is the equilibrium measure for the spectrum and that likely persists at critical coupling.

It seems to be at least possible that the zeros for the critical almost Mathieu model are quasi-clock spaced but with $O\left(n^{-\alpha}\right)(\alpha>1)$ spacing in the bulk.

\section{References}

[1] A. I. Aptekarev, Asymptotic properties of polynomials orthogonal on a system of contours, and periodic motions of Toda chains, Math. USSR Sb. 53 (1986), 233-260; Russian original in Mat. Sb. (N.S.) 125(167) (1984), 231-258.

[2] A. Avila, Y. Last, and B. Simon, Bulk universality and clock spacing of zeros for ergodic Jacobi matrices with a.c. spectrum, preprint.

[3] J. Bellissard, P. D. Hislop, and G. Stolz, Correlation estimates in the Anderson model, J. Stat. Phys. 129 (2007), 649-662.

[4] M. J. Cantero, L. Moral, and L. Velázquez, Measures and para-orthogonal polynomials on the unit circle, East J. Approx. 8 (2002), 447-464.

[5] J. S. Christiansen, B. Simon, and M. Zinchenko, Finite gap Jacobi matrices, I. The isospectral torus, to appear in Constr. Approx.

[6] J. M. Combes, F. Germinet, and A. Klein, Generalized eigenvalue-counting estimates for the Anderson model, J. Stat. Phys. 135 (2009), 201-216.

[7] J. M. Combes, F. Germinet, and A. Klein, Poisson statistics for eigenvalues of continuum random Schrödinger operators, preprint.

[8] E. B. Davies and B. Simon, Eigenvalue estimates for non-normal matrices and the zeros of random orthogonal polynomials on the unit circle, J. Approx. Theory 141 (2006), 189-213.

[9] P. Deift, T. Kriecherbauer, K. T-R McLaughlin, S. Venakides, and X. Zhou, Strong asymptotics of orthogonal polynomials with respect to exponential weights, Comm. Pure Appl. Math. 52 (1999), 1491-1552.

[10] P. Erdös and P. Turán, On interpolation. III. Interpolatory theory of polynomials, Annals of Math. (2) 41 (1940), 510-553.

[11] E. Findley, Universality and zero spacing under local Szegö condition, in preparation.

[12] G. Freud, Orthogonal Polynomials, Pergamon Press, Oxford-New York, 1971.

[13] G. M. Graf and A. Vaghi, A remark on an estimate by Minami, Lett. Math. Phys. 79 (2007), 17-22.

[14] R. Killip and I. Nenciu, Matrix models for circular ensembles, Int. Math. Res. Not. 2004, (2004), 2665-2701.

[15] R. Killip and M. Stoiciu, Eigenvalue statistics for CMV matrices: From Poisson to clock via circular beta ensembles, Duke Math. J. 146 (2009), 361-399.

[16] W. Kirsch and B. Metzger, The integrated density of states for random Schrödinger operators, in "Spectral Theory and Mathematical Physics: A Festschrift in honor of Barry Simon's 60th birthday," pp. 649-696, Proc. Sympos. Pure Math., 76.2, American Mathematical Society, Providence, RI, 2007.

[17] A. Kiselev, Y. Last, and B. Simon, Modified Prüfer and EFGP transforms and the spectral analysis of one-dimensional Schrödinger operators, Comm. Math. Phys. 194 (1998), 1-45. 
[18] S. Kotani, Ljapunov indices determine absolutely continuous spectra of stationary random one-dimensional Schrödinger operators, in "Stochastic Analysis" (Katata/Kyoto, 1982), pp. 225-247, North-Holland Math. Library, 32, North-Holland, Amsterdam, 1984.

[19] S. Kotani, Generalized Floquet theory for stationary Schrödinger operators in one dimension, Chaos Solitons Fractals 8 (1997), 1817-1854.

[20] Y. Last, Almost everything about the almost Mathieu operator, I, in "XIth Internat. Cong. Math. Phys." (Paris, 1994), pp. 366-372, International Press, Cambridge, Mass., 1995.

[21] Y. Last and B. Simon, Fine structure of the zeros of orthogonal polynomials, IV. A priori bounds and clock behavior, Comm. Pure Appl. Math. 61 (2008), 486-538.

[22] E. Levin and D. S. Lubinsky, Applications of universality limits to zeros and reproducing kernels of orthogonal polynomials, J. Approx. Theory 150 (2008), 69-95.

[23] E. Levin and D. S. Lubinsky, Some equivalent formulations of universality limits in the bulk, to appear in Proc. IWOPA'08.

[24] D. S. Lubinsky, A new approach to universality at the edge of the spectrum, Contemp. Math. 458 (2008), 281-290.

[25] D. S. Lubinsky, Universality limits in the bulk for arbitrary measures on compact sets, J. Anal. Math. 106 (2008), 373-394.

[26] D. S. Lubinsky, Universality limits at the hard edge of the spectrum for measures with compact support, Int. Math. Res. Not. 2008 (2008), article ID: rnn099.

[27] D. S. Lubinksy, A new approach to universality limits involving orthogonal polynomials, to appear in Annals of Math.

[28] N. G. Makarov, Metric properties of harmonic measure, Proc. Internat. Cong. Math., 1, 2 (Berkeley, Calif., 1986), pp. 766-776, American Mathematical Society, Providence, RI, 1987.

[29] N. G. Makarov, Fine structure of harmonic measure, St. Petersburg Math. J. 10 (1999), 217-268; Russian original in Algebra i Analiz 10 (1998), 1-62.

[30] A. Máté, P. Nevai, and V. Totik, Szegö's extremum problem on the unit circle, Annals of Math. 134 (1991), 433-453.

[31] N. Minami, Local fluctuation of the spectrum of a multidimensional Anderson tight binding model, Comm. Math. Phys. 177 (1996), 709-725.

[32] S. A. Molchanov, The local structure of the spectrum of the one-dimensional Schrödinger operator, Comm. Math. Phys. 78 (1980/81), 429-446.

[33] P. Nevai, Géza Freud, orthogonal polynomials and Christoffel functions. A case study, J. Approx. Theory 48 (1986), $167 \mathrm{pp}$.

[34] F. Peherstorfer and P. Yuditskii, Asymptotics of orthonormal polynomials in the presence of a denumerable set of mass points, Proc. Amer. Math. Soc. 129 (2001), 3213-3220.

[35] B. Simon, Kotani theory for one dimensional stochastic Jacobi matrices, Comm. Math. Phys. 89 (1983), 227-234.

[36] B. Simon, Orthogonal Polynomials on the Unit Circle, Part 1: Classical Theory, AMS Colloquium Publications, 54.1, American Mathematical Society, Providence, RI, 2005.

[37] B. Simon, Orthogonal Polynomials on the Unit Circle, Part 2: Spectral Theory, AMS Colloquium Series, 54.2, American Mathematical Society, Providence, RI, 2005.

[38] B. Simon, Fine structure of the zeros of orthogonal polynomials, I. A tale of two pictures, Electron. Trans. Numer. Anal. 25 (2006), 328-368.

[39] B. Simon, Fine structure of the zeros of orthogonal polynomials, II. OPUC with competing exponential decay, J. Approx. Theory 135 (2005), 125-139.

[40] B. Simon, Fine structure of the zeros of orthogonal polynomials, III. Periodic recursion coefficients, Comm. Pure Appl. Math. 59 (2006), 1042-1062.

[41] B. Simon, Equilibrium measures and capacities in spectral theory, Inverse Problems and Imaging 1 (2007), 713-772.

[42] B. Simon, Fine structure of the zeros of orthogonal polynomials: A review, in "Difference Equations, Special Functions and Orthogonal Polynomials," pp. 636-653, World Scientific, Singapore, 2007.

[43] B. Simon, Rank one perturbations and the zeros of paraorthogonal polynomials on the unit circle, J. Math. Anal. Appl. 329 (2007), 376-382.

[44] B. Simon, The Christoffel-Darboux kernel, in "Perspectives in PDE, Harmonic Analysis and Applications," pp. 295-335, Proc. Sympos. Pure Math., 79, American Mathematical Society, Providence, RI, 2008. 
[45] B. Simon, Two extensions of Lubinsky's universality theorem, J. Anal. Math. 105 (2008), $345-362$.

[46] B. Simon, Szegö's Theorem and Its Descendants: Spectral Theory for $L^{2}$ Perturbations of Orthogonal Polynomials, in preparation; to be published by Princeton University Press.

[47] H. Stahl and V. Totik, General Orthogonal Polynomials, in "Encyclopedia of Mathematics and Its Applications," 43, Cambridge University Press, Cambridge, 1992.

[48] M. Stoiciu, The statistical distribution of the zeros of random paraorthogonal polynomials on the unit circle, J. Approx. Theory 39 (2006), 29-64.

[49] V. Totik, Asymptotics for Christoffel functions for general measures on the real line, J. Anal. Math. 81 (2000), 283-303.

[50] V. Totik, Polynomial inverse images and polynomial inequalities, Acta Math. 187 (2001), 139-160.

[51] V. Totik, Universality and fine zero spacing on general sets, in preparation.

[52] $\mathrm{H}$. Widom, Extremal polynomials associated with a system of curves in the complex plane, Adv. in Math. 3 (1969), 127-232.

[53] M.-W. L. Wong, First and second kind paraorthogonal polynomials and their zeros, J. Approx. Theory 146 (2007), 282-293.

Mathematics 253-37, California Institute of Technology, Pasadena, CA 91125, USA E-mail address: bsimon@caltech.edu 